\title{
Quercetin enhances adriamycin cytotoxicity through induction of apoptosis and regulation of mitogen-activated protein kinase/extracellular signal-regulated kinase/c-Jun N-terminal kinase signaling in multidrug-resistant leukemia K562 cells
}

\author{
FANG-YUAN CHEN $^{1}$, LAN-FANG CAO ${ }^{2}$, HAI-XIA WAN ${ }^{1}$, MIN-YUE ZHANG $^{1}$, JIA-YI CAI ${ }^{1}$, \\ LI-JING SHEN ${ }^{1}$, JI-HUA ZHONG ${ }^{1}$ and HUA ZHONG ${ }^{1}$ \\ Departments of ${ }^{1}$ Hematology and ${ }^{2}$ Pediatrics, Renji Hospital, School of Medicine, \\ Shanghai Jiao Tong University, Shanghai 200127, P.R. China
}

Received December 26, 2013; Accepted September 29, 2014

DOI: $10.3892 / \mathrm{mmr} .2014 .2734$

\begin{abstract}
Multidrug resistance (MDR) has become a significant challenge in chemotherapeutic treatment of cancer. Quercetin, a naturally occurring flavonoid, has been found to possess anti-proliferative, anti-inflammatory and immunoregulatory bioactivities. The present study was performed to examine the effect of quercetin on human leukemic MDR K562/adriamycin (ADR) cells. Treatment of K562/ADR cells with a combination of quercetin and ADR resulted in potentiation of cytotoxicity, which was measured using a cell counting kit-8 assay. Flow cytometric analysis revealed that quercetin dose-dependently promoted cell apoptosis and treatment with a combination of quercetin and ADR caused synergistic enhancement of the apoptotic effect. In addition, treatment of K562/ADR cells with quercetin alone or in combination with ADR resulted in loss of mitochondrial membrane potential, activation of caspase- $8,-9$ and -3 , reduced expression of the anti-apoptotic proteins B-cell lymphoma (Bcl)-2 and Bcl-extra large and enhanced expression of the pro-apoptotic proteins $\mathrm{Bcl}-2$-interacting mediator of cell death, Bcl-2-associated death promoter and Bcl-2-associated X protein in the cells. Furthermore, the combined treatment of quercetin and ADR synergistically increased the expression of phosphorylated (p-)c-Jun N-terminal kinase and p-p38 mitogen-activated protein kinase and decreased the expression of $\mathrm{p}$-extracellular signal-regulated kinase in the K562/ADR cells. In addition, the expression of P-glycoprotein was significantly decreased following treatment with quercetin alone or in combination with ADR. These findings demonstrated that quercetin is
\end{abstract}

Correspondence to: Dr Lan-Fang Cao, Department of Pediatrics, Renji Hospital, School of Medicine, Shanghai Jiao Tong University, Shanghai 200127, P.R. China

E-mail: cfy62@shsmu.edu.cn

Key words: quercetin, human leukemia, multidrug resistance important in MDR and may be developed into a new reversal agent for cancer chemotherapy.

\section{Introduction}

Multidrug resistance (MDR) refers to the resistance of cancer cells to multiple anticancer drugs and is now a significant clinical challenge facing cancer chemotherapy $(1,2)$. P-glycoprotein (P-gp) is a member of the ATP-binding cassette family, which exports compounds from cells through a process driven by adenosine triphosphate hydrolysis (3). Several chemotherapeutic drugs are known to exert anticancer activity by inducing apoptosis. However, MDR tumor cells are generally resistant to the induction of apoptosis and the resistance of leukemic cells to chemotherapy-induced apoptosis remains the most serious problem in the treatment of leukemia $(4,5)$.

Currently, the preferred strategy for overcoming MDR is to use sensitizer or reversal agents, which are combined with chemotherapeutic drugs (6). Plants have been utilized as medicines and they are an important source of the mainstream pharmacopoeia (Ciwujia, Chinese Pharmacopoeia, 2010: 192-193). There has been substantial effort to identify reversal agents from natural products, which has resulted in significant success $(7,8)$. Flavonoids, which are polyphenolic compounds, are a class of plant secondary metabolites possessing a broad spectrum of pharmacological activities, including anticancer, antimicrobial and immunoregulatory activities (9-11). Quercetin is a naturally occurring flavonoid with a broad spectrum of bioactivities, including antiproliferative, anti-inflammatory and antioxidant effects and effects on the immune system $(12,13)$. Quercetin can inhibit intestinal crypt cell proliferation and aberrant crypt formation, by targeting cyclins and cyclin-dependent kinases (14). Increasing evidence suggests that quercetin can be effective in cancer treatment by inducing cell apoptosis (15), however the function of quercetin on MDR in human leukemia remains to be elucidated. Therefore, the present study aimed to investigate the effect of quercetin on human leukemic MDR K562/adriamycin (ADR) cells. 


\section{Materials and methods}

Reagents. Quercetin and ADR were purchased from Sigma (St. Louis, MO, USA). RPMI-1640 culture medium, fetal bovine serum (FBS), phosphate-buffered saline (PBS), penicillin-streptomycin and $0.25 \%(\mathrm{w} / \mathrm{v})$ trypsin $/ 1 \mathrm{mM}$ ethylenediaminetetraacetic acid were purchased from Gibco-BRL (Grand Island, NY, USA).

Cell culture. K562/ADR human leukemia cells, which were provided by the Chinese Medical Science Research Institute of Hematology (Tian Jin, China), were maintained in RPMI-1640 medium containing $10 \%(\mathrm{v} / \mathrm{v}) \mathrm{FBS}, 100 \mathrm{U} / \mathrm{ml}$ penicillin and $100 \mu \mathrm{g} / \mathrm{ml}$ streptomycin at $37^{\circ} \mathrm{C}$ in a humidified $5 \% \mathrm{CO}_{2}$ incubator. The K562/ADR cells were cultured in the medium containing $1 \mu \mathrm{g} / \mathrm{ml}$ ADR to maintain MDR phenotype and were maintained in drug-free medium for at least 2 days prior to use.

Cell proliferation assay. A cell counting kit-8 (CCK-8) assay was performed for the analysis of cell proliferation. Briefly, the cells $\left(2 \times 10^{5} / \mathrm{ml}\right)$ were seeded into 6 -well plates and left to adhere overnight. The cells were then incubated for 12, 24 and $48 \mathrm{~h}$ at $37^{\circ} \mathrm{C}$ with different concentrations of quercetin and ADR. Subsequently, $20 \mu \mathrm{l}$ CCK-8 was added prior to incubation in the dark at $37^{\circ} \mathrm{C}$ for $4 \mathrm{~h}$. The absorbance was determined using a microplate reader (Thermo Fisher Scientific, Waltham, MA, USA). The concentration of compound required for the proliferation of $50 \%$ of cells to be inhibited $\left(\mathrm{IC}_{50}\right)$ was determined by plotting the percentage of cell growth inhibition against the compound concentration.

Apoptotic assay. The K562/ADR cells were seeded into 12 -well plates and treated with different concentrations of quercetin and ADR, as indicated, for $48 \mathrm{~h}$. The apoptotic morphology of the cells was evaluated by staining with hematoxylin and eosin and visualizing under a light microscope (Leica Microsystems, Wetzlar, Germany; magnification, $\mathrm{x} 200)$. The cells undergoing apoptosis were assessed using an Annexin V-fluorescein isothiocyanate/ propidium iodide (PI) apoptosis detection kit according to the manufacturer's instructions (Becton-Dickinson, Franklin Lakes, NJ, USA). The number of apoptotic cells were quantified using a flow cytometer (FACSCalibur; BD Biosciences, San Jose, CA, USA) and analyzed using CellQuest software (BD Biosciences).

Determination of mitochondrial membrane potential. Changes in mitochondrial transmembrane potential were measured following staining with rhodamine-123. The cells were incubated with the indicated doses of quercetin $(100 \mu \mathrm{g} / \mathrm{ml})$, ADR $(12 \mu \mathrm{g} / \mathrm{ml})$ and quercetin $(100 \mu \mathrm{g} / \mathrm{ml})$ with ADR $(12 \mu \mathrm{g} / \mathrm{ml})$ for $48 \mathrm{~h}$. Rhodamine-123 $(2 \mu \mathrm{l} ; 100 \mu \mathrm{g} / \mathrm{ml})$ was added $1 \mathrm{~h}$ prior to termination of the experiment and the cells were collected and washed in PBS. The fluorescence intensity was then analyzed using a FACSCalibur flow cytometer (Becton-Dickinson).

Western blot analysis. For the western blot analysis of the total cell lysates, the cells were harvested and washed with ice-cold PBS. The protein concentration in the lysates was measured using a bicinchoninic acid protein assay kit (Thermo Fisher Scientific) according to the manufacturer's instructions. Samples of cell lysate (50 $\mu \mathrm{g} / \mathrm{lane})$ were separated using 10\% SDS-PAGE and were then transferred onto polyvinylidene difluoride membranes (Millipore, Billerica, MA, USA). The membranes were incubated overnight at $4^{\circ} \mathrm{C}$ with the following antibodies: B-cell lymphoma (Bcl)-2-interacting mediator of cell death [(BIM; cat. no. 2933, clone C34C5, rabbit monoclonal antibody (mAb)], Bcl-2-associated death promoter (BAD; cat. no. 9239, clone D24A9, rabbit $\mathrm{mAb}$ ), Bcl-2-associated $\mathrm{X}$ protein (BAX; cat. no. 5023, clone D2E11, rabbit mAb), Bcl-extra (xL; cat. no. 2764 , clone $54 \mathrm{H} 6$, rabbit $\mathrm{mAb}$ ), Bcl-2 (cat. no. 2827 , clone 50E3, rabbit mAb), c-Jun N-terminal kinase (JNK; cat. no. 9258, clone 56G8, rabbit $\mathrm{mAb}$ ), ), phosphorylated (p-)JNK (Thr183/Tyr185; cat. no. 4668, clone 81E11, rabbit mAb), p38 mitogen-activated protein kinase (MAPK; cat. no. 9212, clone, rabbit mAb), p-p38 MAPK (Thr180/Thr182; cat. no. 4631 , clone $12 \mathrm{~F} 3$, rabbit $\mathrm{mAb}$ ), extracellular-regulated kinase (ERK)1/2 (cat. no. 4695, clone 137F5, rabbit mAb), p-ERK1/2 (cat. no. 4376, clone 20G11, rabbit mAb), caspase-3 (cat. no. 96686, clone 3G2, mouse mAb), caspase-8 (cat. no. 9746 , clone $1 \mathrm{C} 12$, mouse $\mathrm{mAb}$ ) and caspase-9 (cat. no. 9508, clone $\mathrm{C} 9$, mouse $\mathrm{mAb}$ ) (all 1:1,000; all obtained from Cell Signaling Technology, Inc., Danvers, MA, USA). The membranes were washed three times with tris-buffered saline with Tween 20 and incubated for $1 \mathrm{~h}$ at room temperature with the appropriate secondary polyclonal anti-rabbit $\operatorname{IgG}$ HRP-linked antibody (1:3,000; cat.no. 7074; Cell Signaling Technology, Inc.). Immunoreactive bands were then detected using an enhanced chemiluminescence kit a ChemiGenius bioimaging system (Syngene, Frederick, MD, USA). Western blots were quantified by calculating the gray ratio of target protein: $\beta$-actin.

Determination of the expression of P-gp using flow cytometric analysis. The cells were seeded into a 12-well plate at a density of $2 \times 10^{5} / \mathrm{ml}$ and treated with either quercetin $(100 \mu \mathrm{g} / \mathrm{ml})$, ADR $(12 \mu \mathrm{g} / \mathrm{ml})$ or quercetin $(100 \mu \mathrm{g} / \mathrm{ml})$ with ADR $(12 \mu \mathrm{g} / \mathrm{ml})$. The cells were then collected, washed in PBS and fixed using 70\% cold ethanol following incubation with mouse-anti-human P-gp monoclonal antibody (cat. no 340555). Goat-anti-mouse polyclonal fluorescent antibody (cat.no 20010; Biotium, Inc., Hayward, CA, USA) was used as a secondary antibody and flow cytometry was performed to measure the fluorescence intensity (Becton-Dickinson).

Statistical analysis. For statistical analysis, all the data are expressed as the means \pm standard deviation of at least triplicate determinations and statistical analysis was performed using SPSS software (SPSS, Inc., St. Louis, MO, USA). Comparison between groups was made using analysis of variance. $\mathrm{P}<0.05$ was considered to indicate a statistically significant difference.

\section{Results}

Quercetubg and ADR inhibit leukemia cell proliferation. The cytotoxicity of quercetin and ADR in the K562/ADR 

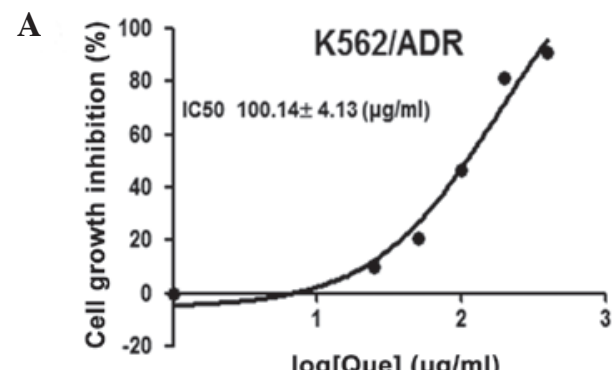

C

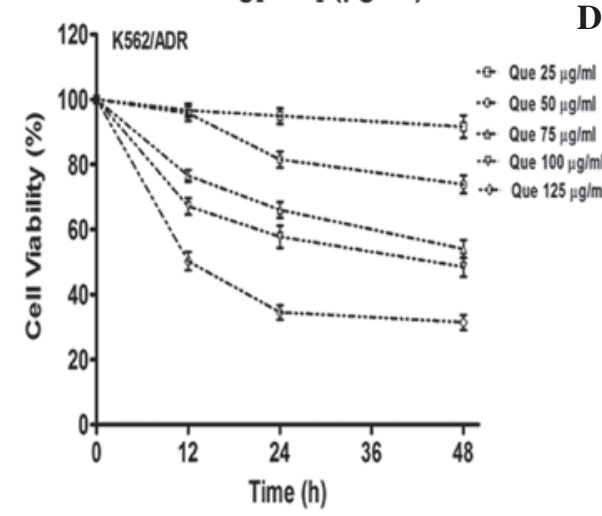

B
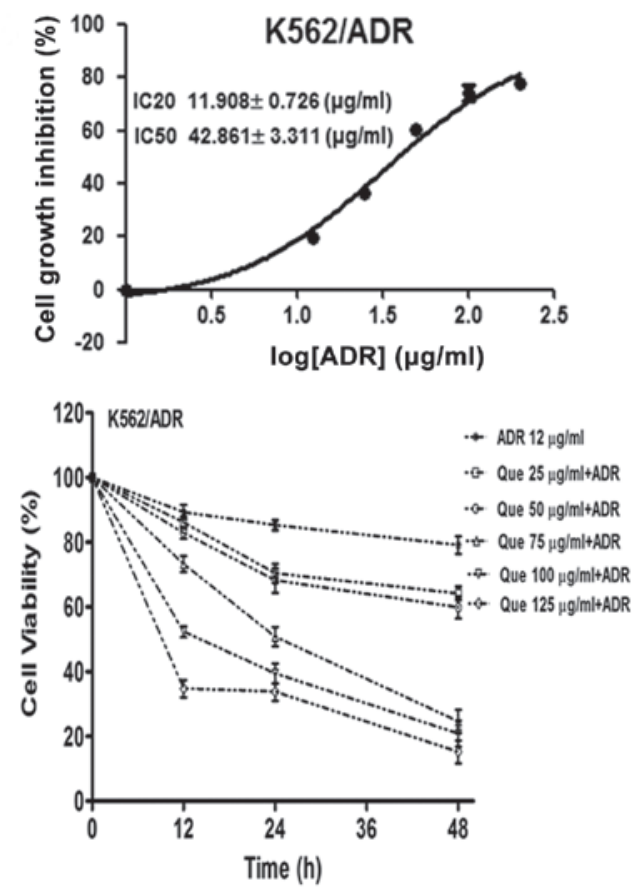

Figure 1. Cytotoxic effect of Que and ADR on leukemia K562/ADR cells. A cell counting kit-8 assay was used to measure the cytotoxicity of (A and C) Que and (B and D) ADR at different concentrations. Data are expressed as the mean \pm standard deviation. Each experiment was repeated at least three times. Que, quercetin; ADR, adriamycin; $\mathrm{IC}_{50}$, half maximal inhibitory concentration; $\mathrm{IC}_{20}$, maximal inhibitory concentration for $20 \%$.

human leukemia cell line was assessed using a CCK-8 assay. The $\mathrm{IC}_{50}$ values of quercetin alone were $100.14 \pm 4.13 \mu \mathrm{g} / \mathrm{ml}$ in the K562/ADR cells following $48 \mathrm{~h}$ of treatment (Fig. 1A). The $\mathrm{IC}_{50}$ and $\mathrm{IC}_{20}$ values of ADR along were $42.86 \pm 3.31$ and $11.91 \pm 0.73 \mu \mathrm{g} / \mathrm{ml}$ in the $\mathrm{K} 562 / A D R$ cells following $48 \mathrm{~h}$ treatment, respectively (Fig. 1B). The CCK-8 assay revealed that quercetin treatment caused concentration-dependent inhibition of cell proliferation (Fig. 1C). In addition, the combination of quercetin and ADR synergistically inhibited cell proliferation (Fig. 1D). Together, these findings demonstrated that treatment of the K562/ADR cells with a combination of quercetin and ADR potentiated the cytotoxicity.

Quercetin and ADR synergistically promote cell apoptosis. Treatment of K562/ADR human leukemia cells for the indicated periods of time with either quercetin and ADR alone or in combination caused nuclear condensation, fragmentation of nuclei and formation of scattered apoptotic bodies, while no clear change in morphology was observed in the nuclei of untreated cells (Fig. 2A). In addition, flow cytometric analysis demonstrated that quercetin promoted cell apoptosis in a concentration- and time-dependent manner (Fig. 2B). Furthermore, a synergistic effect on apoptosis was observed following combination treatment with ADR and quercetin at different concentrations (Fig. 2C).

Loss of mitochondrial membrane potential is induced by quercetin and $A D R$. Mitochondrial damage to cells results in perturbation of mitochondrial membrane potential (16). The present study analyzed the loss in mitochondrial potential in the K562/ADR cells using rhodamine-123 dye. The results demonstrated that $24 \mathrm{~h}$ quercetin treatment, at a concentration of $100 \mu \mathrm{g} / \mathrm{ml}$, resulted in a significant increase in Rh123 fluorescence intensity. The combination of quercetin and ADR further increased the number of Rh123 positive cells. Additionally, a more marked Rh123 positive cell rate was observed following treatment with quercetin alone $(46.60 \pm 1.13 \%)$ and in combination with ADR $(56.08 \pm 0.99 \%)$ after $48 \mathrm{~h}$. Collectively, these results demonstrated that drug combination induced a loss of mitochondrial membrane potential in a synergistic manner (Fig. 3).

Induction of apoptosis signaling cascade by quercetin and $A D R$. Apoptosis can be triggered by extrinsic or intrinsic pathways. The extrinsic pathway involves the cleavage of caspase-8, while the intrinsic apoptotic pathway involves the activation of procaspase-9 (17). Western blot analysis was performed to detect changes in the expression of proteins involved in the apoptotic signaling pathway. The results demonstrated that quercetin and ADR induced the protein expression of a series of caspases, including caspase-8, -9 and -3 (Fig. 4A). The effect of these compounds on the expression of mitochondrial-dependent apoptotic proteins in the K562/ADR cells was also examined. Quercetin and ADR significantly decreased the expression of anti-apoptotic proteins $\mathrm{Bcl}-2$ and $\mathrm{Bcl}-\mathrm{xL}$ and increased the expression of pro-apoptotic proteins Bim, Bad and Bax in the K562/ADR cells (Fig. 4B). Taken together, these findings suggested that quercetin and ADR promoted cell apoptosis by the extrinsic and intrinsic pathways.

Effect of quercetin and ADR on the MAPK/ERK/JNK signaling pathway. To further characterize the mechanisms involved in the pro-apoptotic or proliferation-inhibiting actions of quercetin and ADR, the present study analyzed 

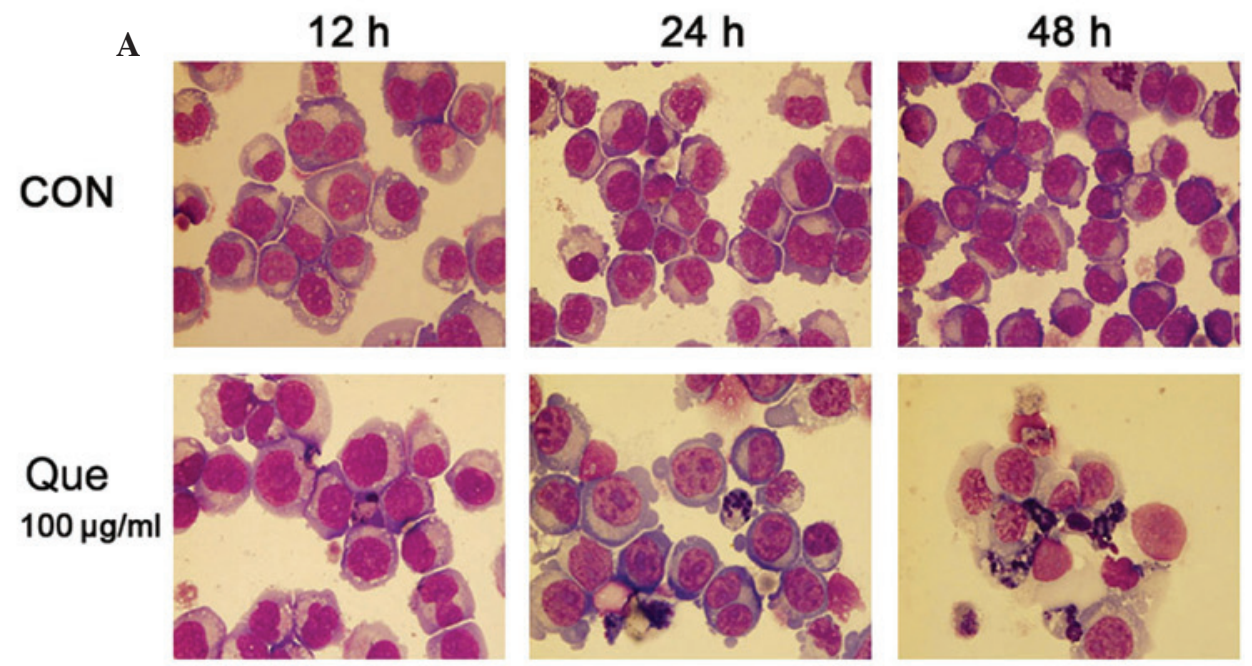

ADR

(IC20)
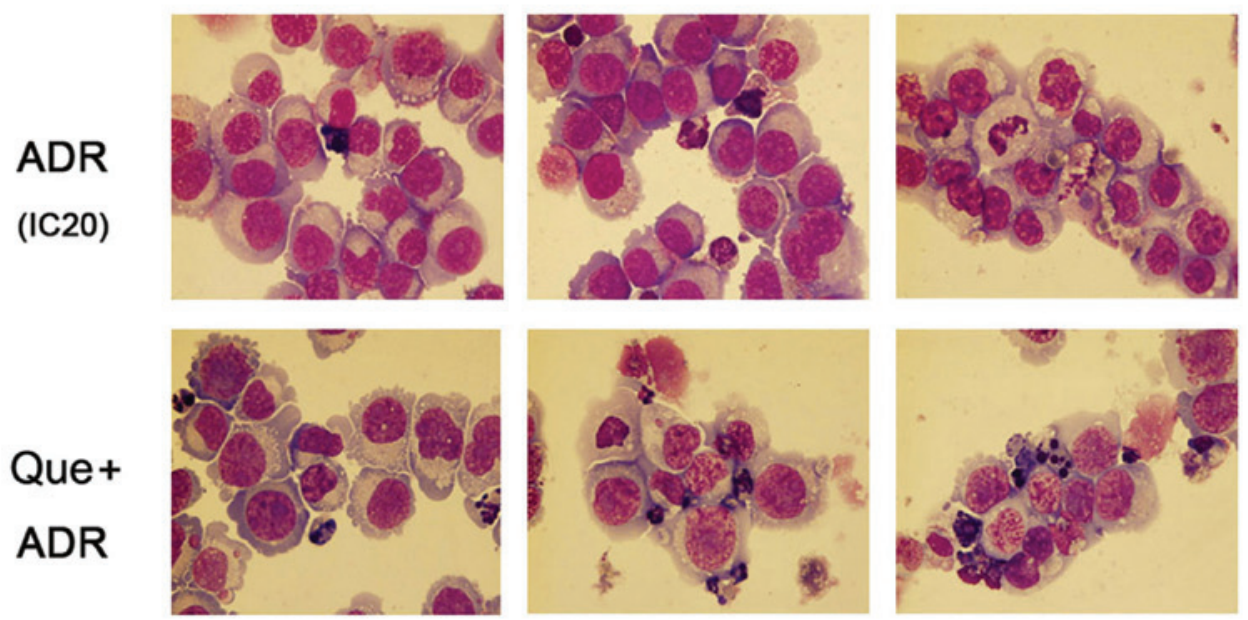

B

$$
\text { C }
$$
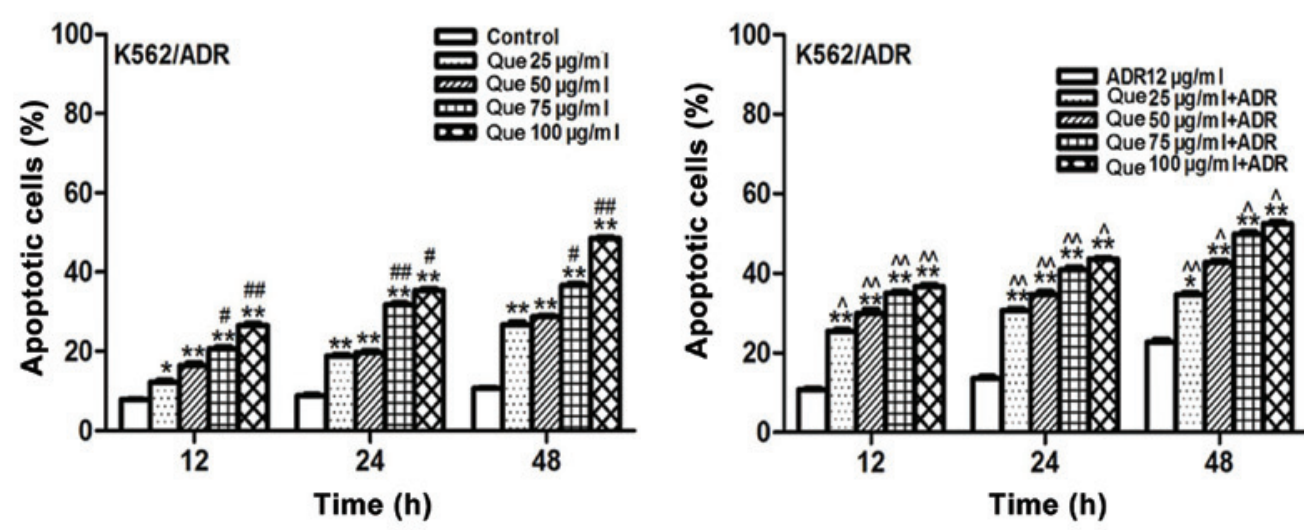

Figure 2. Effect of Que and ADR on K562/ADR cell apoptosis. (A) Changes in cell morphology were observed following treatment with Que or ADR alone or in combination. Flow cytometry was used to analyze the apoptotic rate of the cells following administration with either (B) Que or (C) ADR alone or in combination at different concentrations. ${ }^{*} \mathrm{P}<0.05$ and ${ }^{* *} \mathrm{P}<0.01$, vs. (B) the control or $(\mathrm{C})$ ADR group. ${ }^{*} \mathrm{P}<0.05$ and ${ }^{\# \#} \mathrm{P}<0.01$, compared with treatment with previous concentration of Que at the same time point. ${ }^{\wedge} \mathrm{P}<0.05$ and ${ }^{\wedge} \mathrm{P}<0.01$, ADR (in combination with Que) compared with Que at the same concentration and same time point. Data are expressed as the mean \pm standard deviation. Each experiment was repeated at least three times. Que, quercetin; ADR, adriamycin; $\mathrm{CON}$, control; $\mathrm{IC}_{50}$, half maximal inhibitory concentration; $\mathrm{IC}_{20}$, maximal inhibitory concentration for $20 \%$.

the effect on the main signaling pathways associated with proliferation and the regulation of apoptosis. Western blot analysis was performed to investigate the effect of these compounds on the MAPK/ERK/JNK signaling pathway. The results demonstrated that quercetin and ADR upregulated the content of p-JNK and p-p38 MAPK and downregulated the expression of p-ERK (Fig. 5). These results indicated that the quercetin and ADR-induced cell apoptosis was associated with $\mathrm{MAPK} / \mathrm{ERK} / \mathrm{JNK}$ signaling regulation in the K562/ADR cells.

Effect of quercetin and ADR on the expression of P-gp. In order to examine the reversal effect of quercetin, flow cytometric analysis was performed to measure the expression of 
A

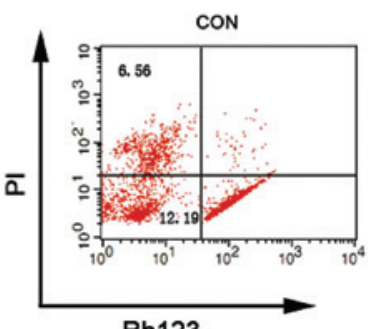

Rh123

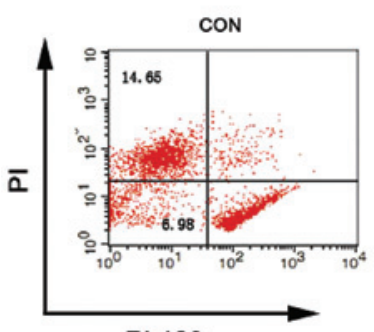

$24 \mathrm{~h}$
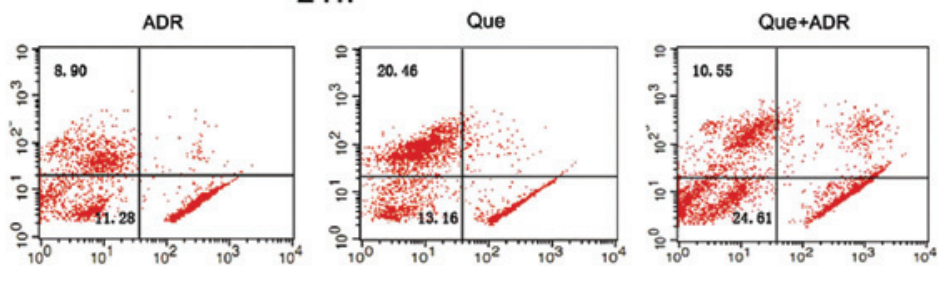

$48 \mathrm{~h}$
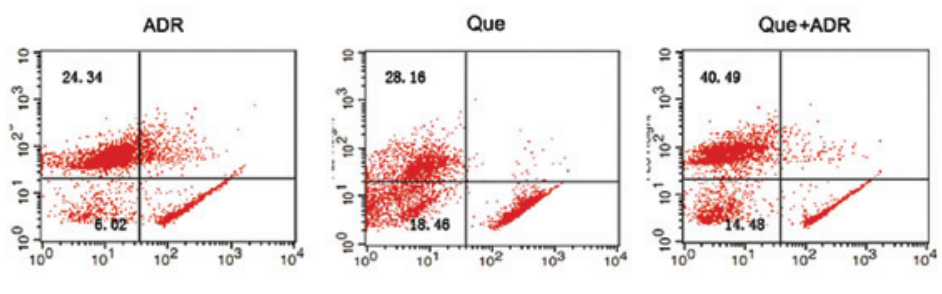

B

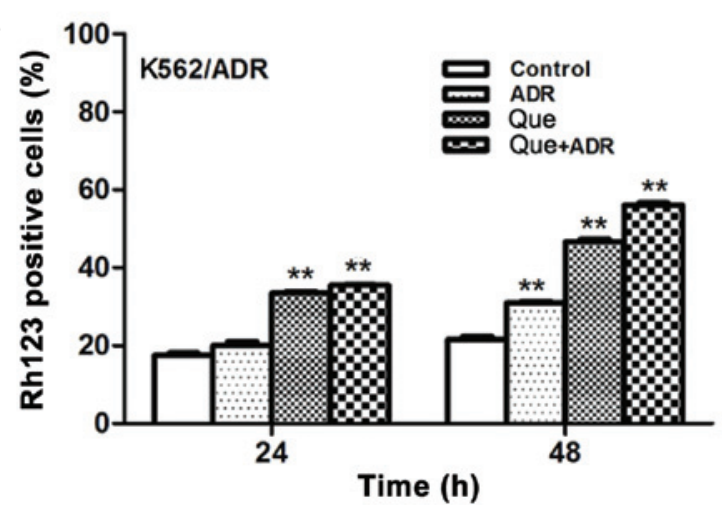

Figure 3. Loss of mitochondrial potential induced by Que and ADR. (A) Following Rh123/PI double staining, flow cytometry was performed to determine changes in mitochondrial membrane potential in the K562/ADR cells treated with Que or ADR, either alone or in combination. (B) Data are expressed as the mean \pm standard deviation. ${ }^{* *} \mathrm{P}<0.01$, compared with the control group. Each experiment was repeated at least three times. Que, quercetin $(100 \mu \mathrm{g} / \mathrm{ml}) ; \mathrm{ADR}$, adriamecin $\left(\mathrm{IC}_{20} ; 12 \mu \mathrm{g} / \mathrm{ml}\right) ; \mathrm{CON}$, control; Rh, rhodamine; PI, propidium iodide.

A
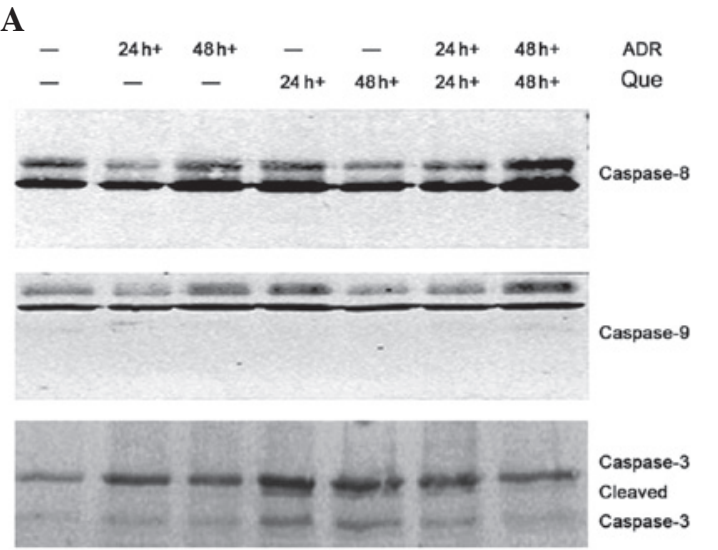

Caspase-3

Cleaved

Caspase-3

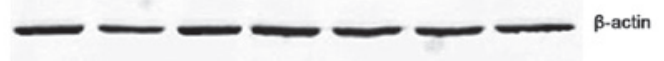

\section{B}

- $24 \mathrm{~h}+48 \mathrm{~h}+\quad-\quad-\quad 24 \mathrm{~h}+48 \mathrm{~h}+\quad$ ADR

$-\quad-\quad-\quad 24 h+48 h+24 h+48 h+$ Que
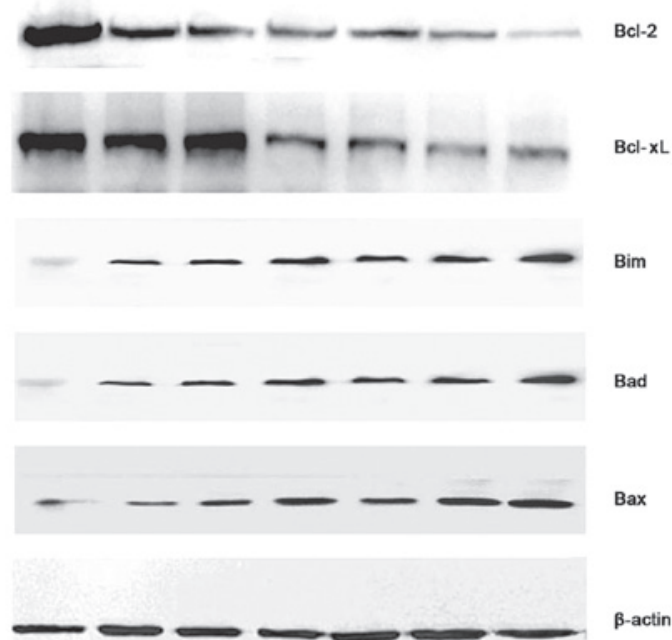

Figure 4. Induction of the apoptotic signaling cascade by Que and ADR. Western blot analysis was performed to determine the expression of (A) caspase-8,-9 and -3 and (B) B-cell lymphoma-2 family members 24 and $48 \mathrm{~h}$ following treatment with either ADR and Que alone or in combination. Results are representative of three separate experiments. $\beta$-actinwas used as a protein loading control. Que, quercetin $(100 \mu / \mathrm{ml}) ; \mathrm{ADR}$, adriamycin (IC $20 ; 12 \mu / \mathrm{ml}) ; \mathrm{Bcl}, \mathrm{B}$-cell lymphoma; $\mathrm{BIM}$. Bcl-2-interacting mediator of cell death; BAD, Bcl-2-associated death promoter; BAX, Bcl-2-associated X protein; Bcl-xL, Bcl-2-extra large. 


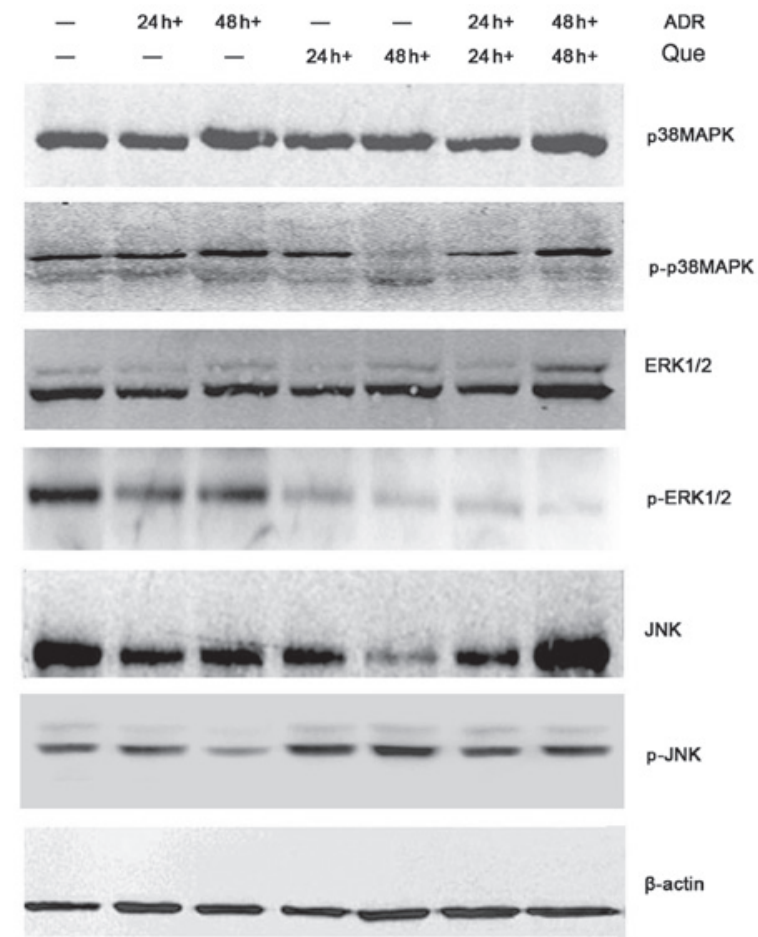

Figure 5. Effect of Que and ADR on the MAPK/ERK/JNK signaling pathway. Western blot analysis was performed to determine the expression of p-JNK, p-p38 MAPK and p-ERK1/2 at 24 and $48 \mathrm{~h}$ following treatment with the indicated drugs. Results are representative of three separate experiments. $\beta$-actin was used as a protein loading control. Que, quercetin; ADR, adriamycin; MAPK, mitogen-activated protein kinase; ERK, extracellular signal-regulated kinase; JNK, c-Jun-N-terminal kinase; p-, phosphorylated.

A

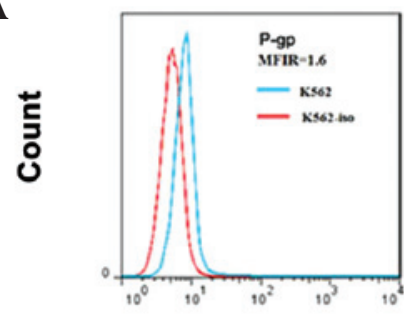

FL2-H

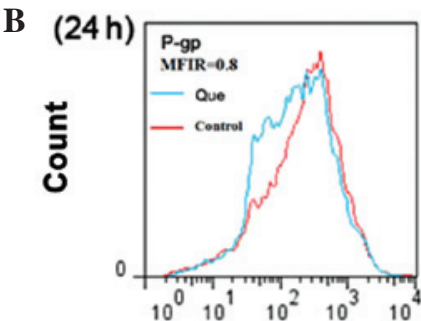

FL2-H

C $(48 h)$

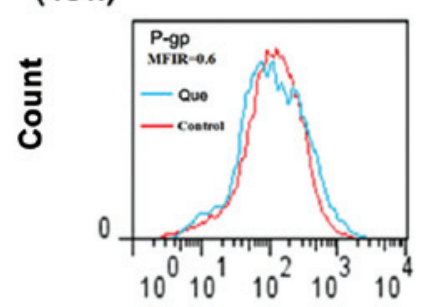

FL2-H

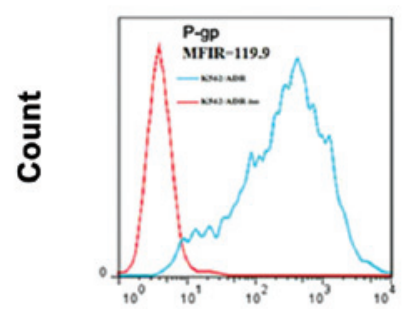

FL2-H

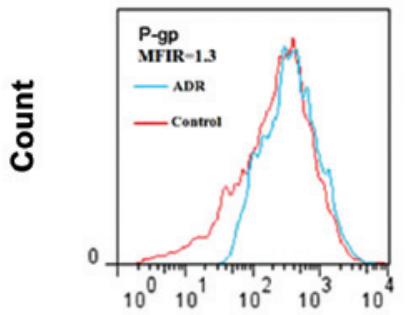

FL2-H

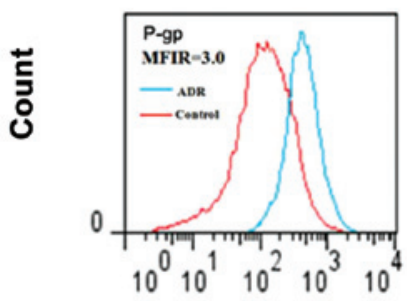

FL2-H

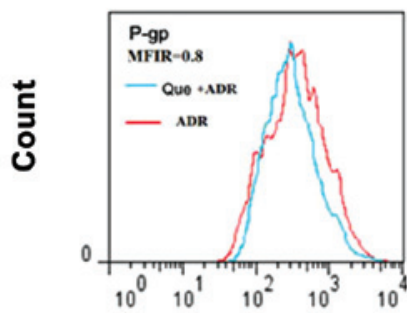

FL2-H

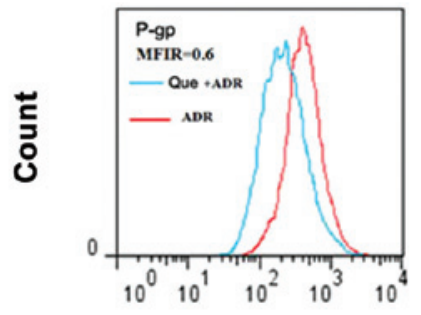

FL2-H

Figure 6. Effect of Que and ADR on the expression of P-gp. (A) Flow cytometry was used to determine the expression of P-gp in the K562/ADR cells prior to and following treatment with Que and ADR at (B) $24 \mathrm{~h}$ and (C) $48 \mathrm{~h}$. Que, quercetin; ADR, adriamycin; P-gp, P-glycoprotein; FL2-H, fluorescence intensity in the FL2 channel; MFIR, mean fluorescence intensity ratio. 
P-gp in the K562/ADR cells. The results indicated that the mean fluorescence intensity ratio in the K562/ADR cells increased significantly (119.9) compared with that in the K562 cells (1.6). However, the expression of P-gp was significantly reduced following $24 \mathrm{~h}$ treatment with either quercetin or ADR, either alone or in combination. The reversal effect on the expression of P-gp was also observed following $48 \mathrm{~h}$ treatment (Fig. 6).

\section{Discussion}

The identification of drugs from medicinal plants has been important in the treatment of cancer. Quercetin is a naturally occurring flavonoid, which has antiproliferative, anti-inflammatory and immunoregulatory activities $(12,13)$. In the present study, the effect and associated mechanism of quercetin on K562/ADR human leukemic MDR cells was investigated. The CCK-8 assay revealed that quercetin had a significant inhibitory effect on the K562/ADR cells in a concentration-dependent manner. The combination of quercetin $(100 \mu \mathrm{g} / \mathrm{ml})$ and ADR $\left(\mathrm{IC}_{20} ; 12 \mu \mathrm{g} / \mathrm{ml}\right)$ resulted in potentiation of the cytotoxicity.

Apoptosis is a complex process, characterized by morphological and biochemical changes in the nucleus and the formation of apoptotic bodies $(18,19)$. The present study observed that quercetin and ADR induced nuclear condensation, fragmentation of nuclei and formation of scattered apoptotic bodies. Flow cytometric analysis also revealed that quercetin promoted cell apoptosis in a concentration- and time-dependent manner and the synergistic effect on apoptosis was more marked following combination treatment with ADR and quercetin at different concentrations.

Apoptosis can be activated by extrinsic or intrinsic signaling pathways (20). The extrinsic pathway involves the cleavage of caspase- 8 , while the intrinsic apoptotic pathway involves procaspase-9, which triggers downstream mitochondrial pro-apoptotic events. Following the activation of initiator caspases, procaspase- 3 becomes activated, which induces cell apoptosis $(21,22)$. In the present study, quercetin and ADR induced the loss of mitochondrial membrane potential. Several studies have suggested that abrogation of mitochondrial membrane potential leads to the activation of caspases $(23,24)$. As demonstrated in the present study, quercetin and ADR led to the activation of a series of caspases, including caspase-8, -9 and -3 in the K562/ADR cells. A marked reduction in the expression levels of anti-apoptotic proteins Bcl-2 and Bcl-xL and increase in the expression levels of pro-apoptotic proteins Bim, Bad and Bax were also observed in the K562/ADR cells. Taken together, these findings suggested that quercetin and ADR promoted cell apoptosis through extrinsic and intrinsic apoptotic pathways in the human leukemia K562/ADR cells.

It has been suggested that the apoptosis of tumor cells involves the activation of JNK and the inactivation of ERK (25). Various studies have demonstrated that activation of the JNK signaling pathway is important in regulation of the expression of pro-apoptotic proteins $(26,27)$. The JNK downstream transcriptional factors phosphorylate the Bcl-2 family members and are involved in various pathophysiological processes, including embryonic development, immune regulation and tumorigenesis $(28,29)$. The results of the present study revealed that quercetin combined with ADR increased the expression of p-JNK and p-p38 MAPK and decreased the expression of p-ERK in a synergistic way, thereby promoting the apoptosis of K562/ADR cells.

Drug resistance in leukemia cells is associated with the increased expression of resistance proteins (30). P-gp, encoded by the MDR 1 gene, is one of the MDR-associated proteins (31). The present study found that quercetin combined with ADR markedly reduced the expression of P-gp.

In conclusion, the findings of the present study suggested that the combination of quercetin and ADR in MCF-7/ADR cells inhibited cell proliferation, promoted apoptosis via regulation in MAPK/ERK/JNK signaling and decreased the expression of P-gp. Therefore, quercetin is of important clinical significance in the MDR of tumor therapy and may be developed into a new reversal agent for cancer chemotherapy.

\section{Acknowledgements}

The authors would like to thank the all members of the Leukemia Research Institute in Renji Hospital. This study was supported by the Shanghai Municipal Bureau of Health Major Subject, the Class of Traditional Chinese Medicine (no. ZYSNXD-CC-ZDYJ001) and the TCM Guide Project from the Natural Science Foundation of Shanghai of China (no. 12401906700).

\section{References}

1. Fang S, Zhu W, Zhang Y, Shu Y and Liu P: Paeoniflorin modulates multidrug resistance of a human gastric cancer cell line via the inhibition of $\mathrm{NF}-\kappa \mathrm{B}$ activation. Mol Med Rep 5: 351-356, 2012.

2. Cho S, Lu M, He X, et al: Notch1 regulates the expression of the multidrug resistance gene $\mathrm{ABCC} 1 / \mathrm{MRP} 1$ in cultured cancer cells. Proc Natl Acad Sci USA 108: 20778-20783, 2011.

3. Stępień KM, Tomaszewski M, Tomaszewska J and Czuczwar SJ: The multidrug transporter P-glycoprotein in pharmacoresistance to antiepileptic drugs. Pharmacol Rep 64: 1011-1019, 2012.

4. Assef Y, Rubio F, Coló G, del Mónaco S, Costas MA and Kotsias BA: Imatinib resistance in multidrug-resistant K562 human leukemic cells. Leuk Res 33: 710-716, 2009.

5. Higgins CF: Multiple molecular mechanisms for multidrug resistance transporters. Nature 446: 749-757, 2007

6. Tai DJ, Jin WS, Wu CS, et al: Changes in intracellular redox status influence multidrug resistance in gastric adenocarcinoma cells. Exp Ther Med 4: 291-296, 2012.

7. Sun L, Chen W, Qu L, Wu J and Si J: Icaritin reverses multidrug resistance of HepG2/ADR human hepatoma cells via downregulation of MDR1 and P-glycoprotein expression. Mol Med Rep 8: 1883-1887, 2013.

8. Limtrakul P, Anuchapreeda S and Buddhasukh D: Modulation of human multidrug-resistance MDR-1 gene by natural curcuminoids. BMC Cancer 4: 13, 2004.

9. Maeda J, Roybal EJ, Brents CA, Uesaka M, Aizawa Y, and Kato TA: Natural and glucosyl flavonoids inhibit poly(ADP-ribose) polymerase activity and induce synthetic lethality in BRCA mutant cells. Oncol Rep 31: 551-556, 2014.

10. Kawai Y, Nishikawa T, Shiba Y, et al: Macrophage as a target of quercetin glucuronides in human atherosclerotic arteries: implication in the anti-atherosclerotic mechanism of dietary flavonoids. J Biol Chem 283: 9424-9434, 2008.

11. Hu QF, Zhou B, Huang JM, et al: Cytotoxic oxepinochromenone and flavonoids from the flower buds of Rosa rugosa. J Nat Prod 76: 1866-1871, 2013.

12. Umathe SN, Dixit PV, Kumar V, Bansod KU and Wanjari MM: Quercetin pretreatment increases the bioavailability of pioglitazone in rats: involvement of CYP3A inhibition. Biochem Pharmacol 75: 1670-1676, 2008. 
13. Punithavathi VR and Prince PS: Pretreatment with a combination of quercetin and alpha-tocopherol ameliorates adenosine triphosphatases and lysosomal enzymes in myocardial infarcted rats. Life Sci 86: 178-184, 2010.

14. Gee JM, Hara H and Johnson IT: Suppression of intestinal crypt cell proliferation and aberrant crypt foci by dietary quercetin in rats. Nutr Cancer 43: 193-201, 2002.

15. Duo J, Ying GG, Wang GW and Zhang L: Quercetin inhibits human breast cancer cell proliferation and induces apoptosis via Bcl-2 and Bax regulation. Mol Med Rep 5: 1453-1456, 2012.

16. Wu YM, Xia XY, Pan LJ et al: Evaluation of sperm mitochondrial function using Rh123/PI dual fluorescent staining. Zhonghua Nan Ke Xue 12: 803-806, 2006 (In Chinese).

17. Sun c, Guo XX, Zhu D et al: Apoptosis is induced in cancer cells via the mitochondrial pathway by the novel xylocydine-derived compound JRS-15. Int J, Mol Sci 41: 850-870, 2013.

18. Li T, Kon N, Jiang L, et al: Tumor suppression in the absence of p53-mediated cell-cycle arrest, apoptosis, and senescence. Cell 149: 1269-1283, 2012.

19. Zhang L, Ren X, Alt E, et al: Chemoprevention of colorectal cancer by targeting APC-deficient cells for apoptosis. Nature 464: 1058-1061, 2010.

20. Tassi E, Zanon M, Vegetti C, et al: Role of Apollon in human melanoma resistance to antitumor agents that activate the intrinsic or the extrinsic apoptosis pathways. Clin Cancer Res 18: 3316-3327, 2012.

21. Franklin EE and Robertson JD: Requirement of Apaf-1 for mitochondrial events and the cleavage or activation of all procaspases during genotoxic stress-induced apoptosis. Biochem J 405: 115-122, 2007.

22. Tsuruma K, Nakagawa T, Morimoto N, et al: Glucocorticoid modulatory element-binding protein 1 binds to initiator procaspases and inhibits ischemia-induced apoptosis and neuronal injury. J Biol Chem 281: 11397-11404, 2006.
23. Li J, Li PF, Dietz R and von Harsdorf R: Intracellular superoxide induces apoptosis in VSMCs: role of mitochondrial membrane potential, cytochrome $\mathrm{C}$ and caspases. Apoptosis 7: 511-517, 2002.

24. Yu CY, Chiang RL, Chang TH, Liao CL and Lin YL: The interferon stimulator mitochondrial antiviral signaling protein facilitates cell death by disrupting the mitochondrial membrane potential and by activating caspases. J Virol 84: 2421-2431, 2010.

25. Kumar A, Byun HS, Bittman R and Saba JD: The sphingolipid degradation product trans-2-hexadecenal induces cytoskeletal reorganization and apoptosis in a JNK-dependent manner. Cell Signal 23: 1144-1152, 2011

26. Lei K and Davis RJ: JNK phosphorylation of Bim-related members of the Bcl2 family induces Bax-dependent apoptosis. Proc Natl Acad Sci USA 100: 2432-2437, 2003.

27. Ha Thi HT, Lim HS, Kim J, Kim YM, Kim HY and Hong S: Transcriptional and post-translational regulation of Bim is essential for TGF- $\beta$ and TNF- $\alpha$-induced apoptosis of gastric cancer cell. Biochim Biophys Acta 1830: 3584-3592, 2013.

28. Kim EK and Choi EJ: Pathological roles of MAPK signaling pathways in human diseases. Biochim Biophys Acta 1802: 396-405, 2010

29. Platanias LC: Map kinase signaling pathways and hematologic malignancies. Blood 101: 4667-4679, 2003.

30. Niedermeier M, Hennessy BT, Knight ZA, et al: Isoform-selective phosphoinositide 3'-kinase inhibitors inhibit CXCR4 signaling and overcome stromal cell-mediated drug resistance in chronic lymphocytic leukemia: a novel therapeutic approach. Blood 113: 5549-5557, 2009.

31. Ebert SP, Wetzel B, Myette RL, et al: Chalcogenopyrylium compounds as modulators of the ATP-binding cassette transporters P-glycoprotein (P-gp/ABCB1) and multidrug resistance protein 1 (MRP1/ABCC1). J Med Chem 55: 4683-4699, 2012. 